\title{
Carboxylic Acid Derivatives of Amlexanox Display Enhanced Potency toward TBK1 and IKK $\varepsilon$ and Reveal Mechanisms for Selective Inhibition ${ }^{\mathbb{1}}$
}

\author{
Tyler S. Beyett, Xinmin Gan, Shannon M. Reilly, Louise Chang, Andrew V. Gomez, \\ Alan R. Saltiel, Hollis D. Showalter, and John J. G. Tesmer \\ Program in Chemical Biology (T.S.B.), Life Sciences Institute (T.S.B., L.C., J.J.G.T.), Departments of Medicinal Chemistry (X.G., \\ H.D.S., J.J.G.T.), Pharmacology (J.J.G.T.), Biological Chemistry (J.J.G.T.), and Vahlteich Medicinal Chemistry Core, College of \\ Pharmacy (X.G., H.D.S.), University of Michigan, Ann Arbor, Michigan; Institute for Diabetes and Metabolic Health (S.M.R., \\ A.V.G., A.R.S.), Departments of Medicine (S.M.R., A.R.S.) and Pharmacology (A.V.G., A.R.S.), University of California, San Diego, \\ La Jolla, California; and Departments of Biological Sciences and of Medicinal Chemistry and Molecular Pharmacology, Purdue \\ University, West Lafayette, Indiana (J.J.G.T.)
}

Received February 15, 2018; accepted August 1, 2018

\section{ABSTRACT}

Chronic low-grade inflammation is a hallmark of obesity, which is a risk factor for the development of type 2 diabetes. The drug amlexanox inhibits $I_{\kappa} \mathrm{B}$ kinase $\varepsilon(\mathrm{IKK} \varepsilon)$ and TANK binding kinase 1 (TBK1) to promote energy expenditure and improve insulin sensitivity. Clinical studies have demonstrated efficacy in a subset of diabetic patients with underlying adipose tissue inflammation, albeit with moderate potency, necessitating the need for improved analogs. Herein we report crystal structures of TBK1 in complex with amlexanox and a series of analogs that modify its carboxylic acid moiety. Removal of the carboxylic acid or mutation of the adjacent Thr156 residue significantly reduces potency toward TBK1, whereas conversion to a short amide or ester nearly abolishes the inhibitory effects. IKK $\varepsilon$ is less affected by these modifications, possibly due to variation in its hinge that allows for increased conformational plasticity. Installation of a tetrazole carboxylic acid bioisostere improved potency to 200 and $400 \mathrm{nM}$ toward IKK $\varepsilon$ and TBK1, respectively. Despite improvements in the in vitro potency, no analog produced a greater response in adipocytes than amlexanox, perhaps because of altered absorption and distribution. The structureactivity relationships and cocrystal structures described herein will aid in future structure-guided inhibitor development using the amlexanox pharmacophore for the treatment of obesity and type 2 diabetes.

\section{Introduction}

Globally, more than two billion people are clinically overweight or obese (Swinburn et al., 2011). Obesity is the leading risk factor for the development of type 2 diabetes and is characterized by chronic, low-grade inflammation in the liver and adipose tissues, which in turn attenuates the actions of multiple hormones to produce a dysregulated metabolic state

This study was supported by National Institutes of Health (NIH) Pharmacological Sciences Training Program fellowship [Grant T32-GM007767] and the US Department of Education GAANN fellowship [Grant P200A150164] to T.S.B., as well as NIH R01 [Grants HL122416, HL071818, DK100319, DK060591, DK60597], K01 [Grant DK105075], and P30 [Grant DK06349]. Use of the Advanced Photon Source was supported by the US Department of Energy, Office of Science, Office of Basic Energy Sciences, under Contract no. DE-AC02-06CH11357, and the use of Life Sciences Collaborative Access Team (LS-CAT) Sector 21 was supported by the Michigan Economic Development Corporation and Michigan Technology Tri-Corridor [Grant 085P1000817].

https://doi.org/10.1124/mol.118.112185.

S This article has supplemental material available at molpharm. aspetjournals.org.
(Mowers et al., 2013; Saltiel, 2016; Reilly and Saltiel, 2017). Feeding mice a high-fat diet results in increased expression of I $\kappa$ B kinase $\varepsilon(\mathrm{IKK} \varepsilon)$ and TANK-binding kinase 1 (TBK1), noncanonical $\mathrm{I} \kappa \mathrm{B}$ kinases that attenuate adrenergic receptormediated thermogenic signaling (Chiang et al., 2009; Mowers et al., 2013) and AMP-activated protein kinase (AMPK) activation (Zhao et al., 2018). The result is a feed-forward mechanism that promotes additional weight gain and further reduces insulin sensitivity.

Most Food and Drug Administration (FDA)-approved therapies for obesity focus on reducing caloric intake or altering the neurologic reward system associated with eating (Saltiel, 2016); however, recognition that inflammation is involved in the progression of obesity and type 2 diabetes has led to an interest in pharmacologically targeting key components of inflammatory pathways. Several years ago, the FDA-approved asthma and aphthous ulcer drug amlexanox (Makino et al., 1987; Bell, 2005) was identified as an inhibitor of IKK $\varepsilon$ and TBK1 (Reilly et al., 2013). When administered to obese mice,

ABBREVIATIONS: AMPK, AMP-activated protein kinase; ANOVA, analysis of variance; DMSO, dimethylsulfoxide; DTT, dithiothreitol; FDA, Food and Drug Administration; $l_{\kappa} \mathrm{B}$, inhibitor of NF- $\kappa$ B; IKK, $I_{\kappa} \mathrm{B}$ kinase; IL-6, interleukin-6; MAPK, mitogen-activated protein kinase; P-loop, phosphatebinding loop; PCR, polymerase chain reaction; PMSF, phenylmethanesulfonylfluoride; qPCR, quantitative polymerase chain reaction; SAR, structure-activity relationship; TBK1, TANK-binding kinase 1; TEV, tobacco etch virus; WT, wild-type. 
catecholamine-stimulated cAMP levels are restored, activating p38 mitogen-activated protein kinase (MAPK) and inducing expression of uncoupling protein 1 (Ucp1) in adipose tissue, which leads to weight loss via increased energy expenditure (Reilly et al., 2015). Insulin sensitivity and regulation of glucose levels were also improved by activation of the hepatic JAK/STAT pathway by interleukin-6 (IL-6), whose expression is regulated by cAMP levels in adipocytes (Reilly et al., 2015). Given the history of safety using amlexanox (Bell, 2005), clinical trials in obese and diabetic patients were conducted in which a subset of patients was identified for their positive response to amlexanox treatment (Oral et al., 2017). These patients showed improvements in hemoglobin A1c, insulin sensitivity, and displayed a reduction in hepatic steatosis while exhibiting changes in energy expenditure gene expression similar to those previously noted in animal studies.

Despite these promising results, the future use of amlexanox in the clinic for obesity and type 2 diabetes intervention will likely be hampered by its limited solubility, as the drug was originally formulated as a topical paste (Bell, 2005), and its moderate potency (Reilly et al., 2013). We demonstrate in vitro that the carboxylic acid on amlexanox strongly contributes to potency against TBK1, whereas its elimination enhances potency toward IKK $\varepsilon$. Replacement of the carboxylic acid with a tetrazole acid bioisostere results in improved potency toward both kinases, whereas conversion to a methyl amide selectively diminished potency toward TBK1, suggesting a route for the development of $\mathrm{IKK} \varepsilon$-selective inhibitors. To understand how amlexanox interacts with this family of kinases and to interpret these results, the cocrystal structure of amlexanox bound to TBK1 was determined. This structure suggested a favorable interaction between the carboxylate on amlexanox and the Thr156 side chain in the active site that likely contributes to the potency of inhibition of IKKe and TBK1. Three additional amlexanox analog cocrystal structures shed light on how carboxylate analogs either improve or diminish potency. Together, these results provide the first insights into the design of improved amlexanox for the treatment of type 2 diabetes and obesity.

\section{Materials and Methods}

Synthesis. Experimental procedures are provided in the Supplemental Material, including literature citations for all synthetic methods used. All starting monomers were obtained from commercial suppliers and used without further purification. Amlexanox (1), >99\% pure by high-performance liquid chromatography, was purchased from Ontario Chemicals, Inc. (Guelph, ON). 2-Amino-7-isopropyl-5oxo-5H-chromeno[2,3-b]pyridine-3-carbonitrile $(8)$ was purchased from Aldrich (St. Louis, MO). Routine ${ }^{1} \mathrm{H}$ nuclear magnetic resonance spectra were recorded at 400 or $500 \mathrm{MHz}$ on Varian 400 or 500 instruments (Varian Inc, Palo Alto, CA), respectively, with chloroform- $d$ or dimethylsulfoxide (DMSO)- $d_{6}$ as solvent. Chemical shift values are recorded in $\delta$ units (parts per million). Mass spectra were recorded on a Micromass TofSpec-2E Matrix-Assisted, Laser-Desorption, Time-ofFlight Mass Spectrometer (Waters Corp., Milford, MA) in a positive electrospray ionization mode unless otherwise noted. High-resolution mass spectrometry analysis was performed on an Agilent Q-TOF system (Santa Clara, CA). Analytical high-performance liquid chromatography was performed on an Agilent 1100 series instrument with an Agilent Zorbax Eclipse Plus C18 $(4.6 \times 75 \mathrm{~mm}, 3.5-\mu \mathrm{m}$ particle size $)$ column with the gradient $10 \%$ acetonitrile/water (1 minute), $10 \%-90 \%$ acetonitrile/water ( 6 minutes), and $90 \%$ acetonitrile/water ( 2 minutes) flow $=1 \mathrm{ml} \mathrm{min}^{-1}$. Thin-layer chromatography was performed on silica gel gypsum hard layer fluorescent plates $(250 \mu \mathrm{m})$ purchased from Analtech (Newark, DE). Column chromatography was carried out in the flash mode using silica gel (220-240 mesh) purchased from Silicycle (Quebec City, QC). Extraction solutions were dried over anhydrous sodium sulfate before concentration.

Protein Expression and Purification. Human TBK1 and IKK $\varepsilon$ spanning residues 1-657 were cloned into a modified $\mathrm{pFastBac}$ vector $(\mathrm{pH} 7 \mathrm{pFB}$, high-throughput protein laboratory at the University of Michigan) containing a tobacco etch virus (TEV) protease cleavable $\mathrm{N}$ terminal hexahistidine tag via ligation-independent cloning. $\mathrm{Mu}-$ tagenesis was performed using the QuikChange site-directed mutagenesis protocol (Agilent Technologies). Positive clones were verified by Sanger sequencing, the DNA transformed into DH10Bac Escherichia coli, and bacmid containing the gene of interest isolated by isopropanol precipitation. Recombinant baculovirus was prepared by transfecting Sf9 insect cells with purified bacmid and passaging several times to obtain virus of a higher titer. For expression, Sf9 or Hi5 insect cells (Invitrogen Technologies, Carlsbad, CA) at a density of $2 \times 10^{6}$ cells ml ${ }^{-1}$ were infected with baculovirus and harvested after 65-72 hours. Cells were pelleted and flash-frozen in liquid nitrogen.

All protein variants were purified using the following protocol. Cell pellets were thawed and resuspended in lysis buffer containing $20 \mathrm{mM}$ HEPES, $\mathrm{pH}$ 7.5, $100 \mathrm{mM} \mathrm{NaCl}, 40 \mathrm{mM}$ imidazole, $1 \mathrm{mM}$ dithiothreitol (DTT), $0.1 \mathrm{mM}$ phenylmethanesulfonylfluoride (PMSF), leupeptin, and lima bean trypsin protease inhibitor. Resuspended cells were homogenized with a Dounce homogenizer before brief sonication. The lysate was clarified by ultracentrifugation for 1 hour at $>200,000 \mathrm{~g}$. The resulting supernatant was glass-filtered before being slowly flowed through Ni-NTA resin. The resin was washed with lysis buffer, and the protein was eluted with lysis buffer supplemented with an additional $200 \mathrm{mM}$ imidazole. IKK $\varepsilon$ was purified via anion exchange chromatography and eluted with a $\mathrm{NaCl}$ gradient from 0.0 to $1.0 \mathrm{M}$ at $\mathrm{pH} 7.5$ and protein of $>95 \%$ purity by SDS-PAGE concentrated to $\sim 1 \mathrm{mg} \mathrm{ml}^{-1}$ by absorbance at $280 \mathrm{~nm}$ using the calculated IKK $\varepsilon$ molecular weight and molar extinction coefficient $76,610 \mathrm{kDa}$ and $57,300 \mathrm{M}^{-1} \mathrm{~cm}^{-1}$, respectively, and flash-frozen in liquid nitrogen.

TBK1 was further purified by anion-exchange chromatography using a HiTrap $\mathrm{Q}$ column and eluted with a $\mathrm{NaCl}$ gradient from 0.0 to $1.0 \mathrm{M}$ at $\mathrm{pH}$ 7.5. TBK1 to be used for kinase assays was concentrated to $\sim 1 \mathrm{mg} \mathrm{ml}^{-1}$ by absorbance at $280 \mathrm{~nm}$ using the TBK1 molecular weight and molar extinction coefficient $75,650 \mathrm{kDa}$ and $78,730 \mathrm{M}^{-1} \mathrm{~cm}^{-1}$, respectively, and flash-frozen in liquid nitrogen. TBK1 from anion exchange to be used for crystallography was incubated at $4^{\circ} \mathrm{C}$ overnight with $\sim 10 \%(\mathrm{w} / \mathrm{w})$ TEV protease while being dialyzed against $1 \mathrm{l}$ of $20 \mathrm{mM}$ HEPES, $\mathrm{pH} 7.5,100 \mathrm{mM} \mathrm{NaCl}$, and $1 \mathrm{mM}$ DTT in 6- to $8000-\mathrm{kDa}$ cutoff dialysis tubing to cleave the $6 \mathrm{His}$ tag. The next day, the mixture was passed through Ni-NTA resin to remove TEV protease. The cleaved TBK1 protein was dephosphorylated by incubating at room temperature for 4-6 hours with $\lambda$ phosphatase (New England Biolabs, Ipswich, MA) in buffer supplemented with $1 \mathrm{mM} \mathrm{MnCl}_{2}$. The dephosphorylated protein was further purified via size-exclusion chromatography on an S200 column in buffer lacking $\mathrm{MnCl}_{2}$ (20 mM HEPES, pH 7.5, $100 \mathrm{mM} \mathrm{NaCl}$, and $1 \mathrm{mM}$ DTT). Purified TBK1 for crystallography was concentrated to $\sim 3 \mathrm{mg} \mathrm{ml}^{-1}$ as determined by absorbance at $280 \mathrm{~nm}$ and flash-frozen in liquid nitrogen.

Crystallization and Structure Determination. Crystallization conditions were based on those previously reported with modification (Larabi et al., 2013; Tu et al., 2013). TBK1-amlexanox crystals were grown at $20^{\circ} \mathrm{C}$ via hanging drop vapor diffusion in drops containing $1 \mu \mathrm{l}$ of $0.1 \mathrm{M}$ HEPES, pH 7.5, 4\% (w/v) PEG 8,000, $2 \mu \mathrm{l}$ of purified wild-type (WT) human TBK1 (residues 1-657 at $\sim 3 \mathrm{mg} \mathrm{ml}^{-1}$ ), and $0.2 \mu \mathrm{l}$ amlexanox ( $10 \mathrm{mM}$ in DMSO) over wells containing $1 \mathrm{ml}$ of $0.1 \mathrm{M}$ HEPES pH 7.5, 4\% (w/v) PEG 8,000, and 10\% DMSO. To obtain TBK1 crystals in complex with the ethyl ester (3), tetrazole $(\mathbf{9})$, or amide-coupled tetrazole (5a) analogs of amlexanox, TBK1 was incubated with $1 \mathrm{mM}$ inhibitor (from $100 \mathrm{mM}$ stock in DMSO) for 1 hour on ice. Crystals were grown at $20^{\circ} \mathrm{C}$ via hanging drop vapor diffusion in 
drops containing $1 \mu \mathrm{l}$ of well solution and $1 \mu \mathrm{l}$ of purified WT human TBK1 (residues 1-657 at $\sim 3 \mathrm{mg} \mathrm{ml}^{-1}$ ) over wells containing $1 \mathrm{ml}$ of 0.1 M HEPES, $\mathrm{pH}$ 7.0-8.5, and 1\%-6\% (w/v) PEG 8000. Small diamonds $\leq 25 \mu \mathrm{m}$ appeared within 1 to 2 days and grew for an additional 1-3 days to 50-100 $\mu \mathrm{m}$ in all dimensions. Crystals were harvested and briefly soaked in a cryoprotecting solution composed of 0.1 M HEPES, pH 8.5, 8\% (w/v) PEG 8,000, 30\% (v/v) PEG 400, and $0.5 \mathrm{mM}$ inhibitor before flash-freezing in liquid nitrogen.

Data were collected at Life Sciences Collaborative Access Team (LS-CAT, Advanced Photon Source; Argonne National Laboratory, Lemont, IL) under a cryostream maintained at $100 \mathrm{~K}$ using either a MAR300 or an Eiger 9M detector. All individual software was used as part of the SBGrid package (Morin et al., 2013). Data were processed with XDS (Kabsch, 2010), and the structure was solved in Phenix (Adams et al., 2010) through molecular replacement implemented by Phaser (McCoy, et al., 2007) with a ligand-free structure of TBK1 (PDB 4IM0) as the search model. Models were built by alternating rounds of manual model building in Coot (Emsley et al., 2010) with reciprocal space refinement in Phenix alongside validation with MolProbity (Chen et al., 2010). Ligand restraints were generated with eLBOW in Phenix using the AM1/RM1 semiempirical quantum-mechanical optimization method (Moriarty et al., 2009). Graphics were prepared with PyMol version 1.6 (Schrödinger, LLC, New York, NY). Coordinates and diffraction amplitudes have been deposited in the Protein Data Bank with the accession codes $5 \mathrm{~W} 5 \mathrm{~V}, 6 \mathrm{BNY}, 6 \mathrm{BOD}$, and $6 \mathrm{BOE}$.

Radiometric Dose-Response Assays. Reactions containing $50 \mathrm{nM}$ TBK1 or IKK $\varepsilon$ and $5 \mu \mathrm{M}$ bovine myelin basic protein (MyBP; EMD Millipore, Billerica, MA) in reaction buffer (50 mM HEPES, pH $7.5,10 \mathrm{mM} \mathrm{NaCl}, 10 \mathrm{mM} \mathrm{MgCl}_{2}$, and $1 \mathrm{mM}$ DTT) with varying concentrations of inhibitor were initiated with $5 \mu \mathrm{M}$ ATP spiked with $\left[\gamma_{-}{ }^{32} \mathrm{P}\right]$-ATP (PerkinElmer, Waltham, MA) and allowed to proceed for 30 minutes at room temperature in a final volume of $20 \mu \mathrm{l}$. Reactions were quenched with SDS gel loading dye, run on 4\%-15\% SDS-PAGE gels, and imaged on phosphor screens using a Typhoon imager (GE Healthcare, Chicago, IL). Band intensities corresponding to phosphorylated MyBP were quantified with ImageQuant and the intensities normalized to account for variation in scan intensities. The data were analyzed in GraphPad Prism 7 (GraphPad Software, San Diego, CA) using a three-parameter dose-response curve model with the Hill slope constrained to 1 and automatic outlier rejection. Statistical significance was determined in GraphPad Prism via twoway analysis of variance (ANOVA) with Dunnett's multiple comparison correction relative to amlexanox $(n=3)$.

Thermal Shift Assay. Differential scanning fluorometry was performed in an HT7900 quantitative polymerase chain reaction (PCR) instrument (ThermoFisher, Waltham, MA) using $0.2 \mathrm{mg} \mathrm{ml}^{-1}(2.6 \mu \mathrm{M})$ TBK1 in assay buffer (20 mM HEPES, $\mathrm{pH} 8.0,5 \mathrm{mM} \mathrm{MgCl}_{2}$, and $1 \mathrm{mM}$ DTT), SYPRO orange protein gel stain (1000x stock; Sigma-Aldrich), and $200 \mu \mathrm{M}$ inhibitor. Melt curves were obtained by increasing the temperature from 25 to $60^{\circ} \mathrm{C}$ at a rate of $2^{\circ} \mathrm{C} \mathrm{min}{ }^{-1}$. Data were plotted in GraphPad Prism 7 with the inflection point of the sigmoidal curve representing the melting point $\left(T_{\mathrm{m}}\right)$ of the protein. The experiment was performed three times in duplicate using protein from the same purification. Statistical significance was assessed via two-way ANOVA with Bonferroni correction for multiple comparisons $(n=2)$.

Solubilization of Compounds. Amlexanox (20 mg) is completely dissolved in $0.35 \mathrm{ml}$ of $200 \mathrm{mM} \mathrm{NaOH}$ at room temperature by vibration with ultrasonic apparatus, $0.50 \mathrm{ml}$ of PEG-400 added and shaken, and $0.15 \mathrm{ml}$ of phosphate-buffered saline buffer $(\mathrm{pH}=7.4)$ added, and the mixture is shaken by hand again, resulting in a clear aqueous solution. Analog $\mathbf{9}(2 \mathrm{mg})$ is completely dissolved in $0.62 \mathrm{ml}$ of $10 \mathrm{mM} \mathrm{NaOH}$ at room temperature, and $0.18 \mathrm{ml}$ of $25 \mathrm{mM}$ Tris-HCl buffer $(\mathrm{pH}=7.6)$ is added. Similar attempts to make a $1 \mathrm{mg} / \mathrm{ml}$ solution of 11 under neutral, basic, or acidic conditions were unsuccessful.

3T3-L1 Differentiation. 3T3-L1 fibroblasts (American Type Culture Collection, Manassas, VA) were cultured in Dulbecco's modified
Eagle's medium containing $10 \%$ fetal bovine serum. Once grown to confluence, adipocyte differentiation was initiated using a threecomponent cocktail containing $500 \mu \mathrm{M}$ 3-isobutyl-1-methylxanthine, $250 \mathrm{nM}$ dexamethasone, and $1 \mu \mathrm{g} \mathrm{ml}^{-1}$ insulin for the first 3 days, followed by an additional 3 days in media containing insulin; finally, differentiation was completed in the culture media. Only cultures in which $>90 \%$ of cells displayed adipocyte morphology were used. Fully differentiated adipocytes, which had been cultured in the fetal bovine serum-only media for 1 week, were treated with $100 \mu \mathrm{M}$ amlexanox analog, amlexanox, or vehicle control. Cells were harvested after 1 hour of treatment of Western blot analysis. Media were collected after 4 hours of treatment of IL-6 measurement.

Western Blot Analysis. Cells were homogenized in $50 \mathrm{mM}$ Tris, $\mathrm{pH}$ 7.5, $150 \mathrm{mM} \mathrm{NaCl}, 2 \mathrm{mM}$ EDTA, $10 \%$ glycerol, $1 \%$ Triton X-100, $1 \mathrm{mM}$ DTT, $1 \mathrm{mM} \mathrm{Na}_{3} \mathrm{VO}_{4}, 5 \mathrm{mM} \mathrm{NaF}, 1 \mathrm{mM}$ PMSF, $25 \mathrm{mM}$ glycerol 2-phosphate, and freshly added protease inhibitor tablet, and then incubated them for 1 hour at $4^{\circ} \mathrm{C}$. Cell lysates were produced in an SDS lysis buffer (100 mM Tris, pH 7.5, $130 \mathrm{mM} \mathrm{NaCl}, 1 \% \mathrm{NP}-40,0.1 \%$ SDS, $0.2 \%$ sodium deoxycholate, $1 \mathrm{mM} \mathrm{Na}_{3} \mathrm{VO}_{4}, 1 \mathrm{mM} \mathrm{NaF}, 100 \mathrm{mM}$ $\mathrm{Na}_{4} \mathrm{P}_{2} \mathrm{O}_{7}, 1 \mathrm{mM}$ PMSF, $25 \mathrm{mM}$ glycerol 2-phosphate, and freshly added protease inhibitor tablet) and sonicated for three 5-second pulses at an output power of $6 \mathrm{~W}$. Crude lysates were centrifuged at $14,000 \mathrm{~g}$ for 15 minutes twice, and the protein concentration was determined using Bio-Rad Protein Assay Dye Reagent. Samples were diluted in SDS sample buffer. Bound proteins were resolved by SDSPAGE and transferred to nitrocellulose membranes (Bio-Rad, Hercules, CA). Individual proteins were detected with specific antibodies and visualized on film using horseradish peroxidase-conjugated secondary antibodies (Bio-Rad) and Western lightning-enhanced chemiluminescence (PerkinElmer Life Sciences). Antibodies TBK1 (3013), phosphorylated TBK1 (Ser172-5483), p38 (9212), and phosphorylated p38 (Thr180/Tyr182-9211) were purchased from Cell Signaling Technology (Danvers, MA).

IL-6 Measurement. Il-6 levels were quantified using Il-6 Quantikine enzyme-linked immunoassay (ELISA) from R\&D systems (Minneapolis, MN), with $50 \mu \mathrm{l}$ of cell culture media, and two-way ANOVA with Dunnett's correction for multiple comparisons relative to DMSO control $(n=3)$.

Gene Expression Analysis. RNA extractions from tissues were performed using the RNeasy Lipid Tissue kit (Qiagen, Hilden, Germany). Whereas RNA extraction from cells used the RNeasy Kit, we used the Superscript First-Strand Synthesis system for reverse transcription-PCR (Invitrogen) with a 3:1 mixture of random hexamers/ oligo dT primers. Real-time PCR amplification was performed on samples in triplicate with Power SYBR Green PCR master mix (Applied Biosystems, Foster City, CA) using the Applied Biosystems 7900HT Fast real-time PCR system and quantified using an internal standard curve and Arbp as the control gene. Statistical significance was determined by a two-way ANOVA with Dunnett's correction for multiple comparisons relative to DMSO control $(n=3)$.

\section{Results}

Synthesis of C-3 Derivatives of Amlexanox. We focused on simple modifications of the C-3 carboxylic acid function of amlexanox (1) (Scheme 1) to selected acid bioisosteres or ester and amide analogs to assess their effects on binding to our target enzymes and improving physicochemical properties and pharmacokinetics. Treatment of $\mathbf{1}$ with thionyl chloride and catalytic DMF provided key acid chloride intermediate $\mathbf{2}$, onto which standard reaction conditions were applied to give the known ethyl ester 3 (84\% yield) (Nohara et al., 1985), primary alcohol 4 (78\% yield), and 5-aminotetrazole amide 5a (62\% yield). Simpler amide derivatives were derived directly from amlexanox using standard coupling conditions (EDC, HOBT) to provide analogs $\mathbf{5 b - d}$ in 
<smiles>CC(C)c1ccc2oc3nc(N)c(CO)cc3c(=O)c2c1</smiles>

(c)<smiles>C1CCCCC1</smiles><smiles>CC(C)NCCOC(=O)c1cc2c(=O)c3cc(C(C)C)ccc3oc2nc1N</smiles><smiles>CC(C)c1ccc2oc3nc(N)ccc3c(=O)c2c1</smiles><smiles>CC(C)c1ccc2oc3nc(N)c(C(=O)Cl)cc3c(=O)c2c1</smiles>

2<smiles></smiles>

1

(amlexanox) (b)<smiles>CCOC(=O)c1cc2c(=O)c3cc(C(C)C)ccc3oc2nc1N</smiles>

(d)

$$
\begin{aligned}
& \text { a: } \mathrm{R}=\mathrm{N}_{\mathrm{N}}^{\mathrm{N}} \\
& \text { b: } \mathrm{R}=\mathrm{Me} \\
& \text { c: } \mathrm{R}=-\left(\mathrm{CH}_{2}\right)_{2} \mathrm{NEt}_{2} \\
& \text { d: } \mathrm{R}=-\left(\mathrm{CH}_{2}\right)_{2} \mathrm{~N}-\text { morpholino }
\end{aligned}
$$

Scheme 1. Reagents and conditions: (a) $\mathrm{SOCl}_{2} /$ cat. DMF, DCM, reflux, 4 hours, $\sim 100 \%$. (b) EtOH, reflux, 1 hour, $84 \%$. (c) 4 eq. NaBH 4 , THF, RT, 1.5 hour, 78\%. (d) for 5a: 5-aminotetrazole, pyridine, RT, 7 hours, $62 \%$. (e) for 5b-d: $\mathrm{RNH}_{2}$, EDC.HCl, HOBT, DIEA, DMF, RT, 16 hours, $40 \%-48 \%$. (f) CDI, 2-(dimethylamino)ethanol, DMF, $60^{\circ} \mathrm{C}, 22$ hours, $89 \%$; ethereal HCl, DCM, RT, 85\%. (g) cat. Cu metal, quinolone, reflux, $60 \%$. See Supplemental Material for full names of solvent and reagent abbreviations.

$40 \%-48 \%$ isolated yields. The synthesis of a water-soluble ester derivative was best achieved through formation of the 1-imidazolyl ester of amlexanox, followed by reaction with 2-(dimethylamino)ethanol. Workup followed by hydrochloride salt formation provided $\mathbf{6}$ in $76 \%$ overall yield. To test the effect on the biologic activity of swapping the 3-carboxylic acid function of amlexanox for a hydrogen, analog 7 was derived in $60 \%$ yield using classic methods for the decarboxylation of aromatic compounds (copper, refluxing quinolone).

The synthesis of selected C-3 carboxylic acid bioisosteres of amlexanox is delineated in Scheme 2. Toward this end, analogs 9, 11, and 12 were synthesized using standard methods. The reaction of the commercially available C-3 nitrile of amlexanox $(\mathbf{8})$ with sodium azide provided the known tetrazole $\mathbf{9}$ (Nohara et al., 1985) in $82 \%$ yield. Reaction of this with hydroxylamine afforded carboximidamide intermediate 10, from which ring closure reactions with triphosgene and triethylorthoformate provided the 1,2,4-oxadiazol-5(4H)-one 11 in $78 \%$ yield and 1,2,4-oxadiazole 12 in 62\% yield, respectively. Compound structural assignments are supported by diagnostic peaks in the ${ }^{1} \mathrm{H}$ nuclear magnetic resonance spectra and by mass spectrometry.

Carboxylic Acid on Amlexanox Enhances Potency. Amlexanox displays an in vitro $\mathrm{IC}_{50}$ of $0.8 \pm 0.1$ and $5.8 \pm$ $0.8 \mu \mathrm{M}$ toward TBK1 and IKK $\varepsilon$, respectively (Table 1 ). Two analogs of amlexanox were synthesized in which the carboxylic acid was reduced to a primary alcohol (4) or removed (7). Reduction of the carboxyl in 4 decreased potency 30 -fold, whereas elimination of the carboxylic acid in $\mathbf{7}$ decreased potency 40-fold toward TBK1 (Table 1). Reduction of the carboxylate only modestly reduced potency toward IKK $\varepsilon$, whereas elimination of the group enhanced potency 2 -fold. These data show that the carboxylic acid of amlexanox strongly influences the inhibition of TBK1 but less so in the case of IKK $\varepsilon$.

Amide and Ester Derivatives Reduce Inhibition. Methyl (5b), 2-(diethylamino)ethyl (5c), and morpholine (5d) amide derivatives were synthesized to expand the structureactivity relationship (SAR) around the carboxylic acid moiety. Potency of 5c and 5d toward TBK1 was decreased by 45 -fold<smiles>CC(C)c1ccc2oc3nc(N)c(C#N)cc3c(=O)c2c1</smiles>

(a)<smiles>CC(C)c1ccc2oc3nc(N)c(-c4nn[nH]n4)cc3c(=O)c2c1</smiles>

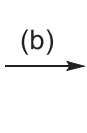<smiles>CC(C)c1ccc2oc3nc(N)c(C(=N)NO)cc3c(=O)c2c1</smiles>

10 (d)<smiles>CC(C)c1ccc2oc3nc(N)c(-c4ncon4)cc3c(=O)c2c1</smiles>

12

Scheme 2. Reagents and conditions: (a) $\mathrm{NaN}_{3}, \mathrm{NH}_{4} \mathrm{Cl}$, DMF, $90^{\circ} \mathrm{C}, 16$ hours, $82 \%$. (b) $\mathrm{NH}_{2} \mathrm{OH} \cdot \mathrm{HCl}$, aq. $\mathrm{NaHCO}_{3}$, EtOH, reflux, $92 \%$. (c) triphosgene, p-dioxane, $100^{\circ} \mathrm{C}, 4$ hours, then $\mathrm{H}_{2} \mathrm{O}, 78 \%$. (d) $(\mathrm{EtO})_{3} \mathrm{CH}$, cat. $\mathrm{BF}_{3} \cdot \mathrm{Et}_{2} \mathrm{O}, 80^{\circ} \mathrm{C}, 1$ hour, $62 \%$. See Supplemental Material for full names of solvent and reagent abbreviations. 
TABLE 1

Potencies of Amlexanox Analogs

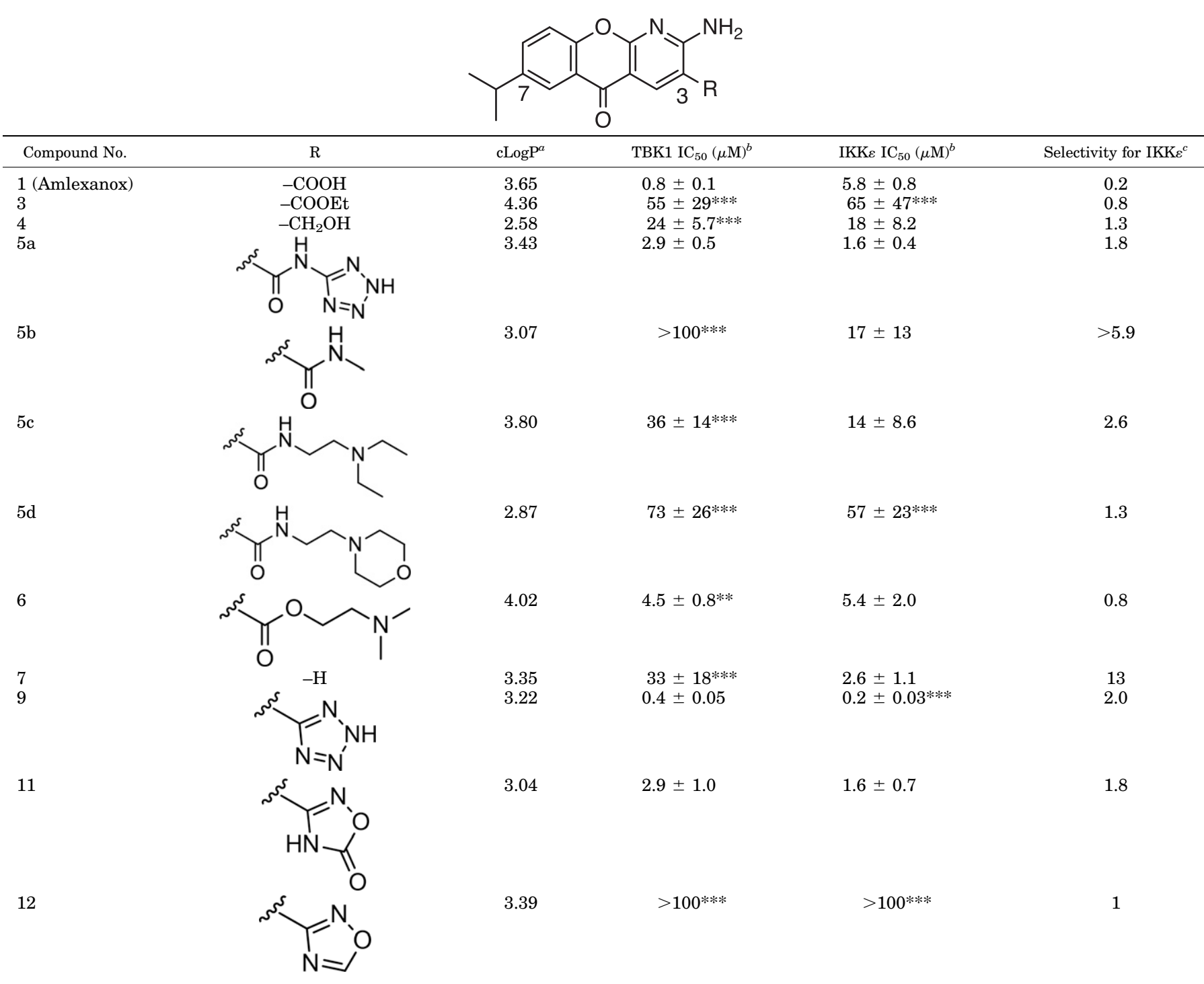

${ }^{a}$ Calculated partitioning coefficient (calculated via Marvin, ChemAxon). Larger values suggest enhanced cellular permeability.

${ }^{b}$ In vitro potency $\left(\mathrm{IC}_{50}\right)$ toward TBK1 and $\operatorname{IKK} \varepsilon(n=3$, mean \pm S.E.M.). Statistical significance from amlexanox determined by two-way ANOVA with Dunnett's multiple comparison correction. $* P \leq 0.05 ; * * P \leq 0.01 ; * * * P \leq 0.001$.

${ }^{c}$ Selectivity determined by diving mean TBK1 $\mathrm{IC}_{50}$ by the mean $\mathrm{IKK} \varepsilon \mathrm{IC}_{50}$.

and 90 -fold, respectively. Similar decreases in potency were observed toward IKK $\mathrm{IK}$. A selective loss in potency of more than $\mathbf{1 2 5}$-fold for TBK1 was observed for $\mathbf{5 b}$. IKK $\varepsilon$ is however more tolerant to short modifications of the carboxylic acid in $\mathbf{5 b}$, which displays only a 3 -fold reduction in potency. A pair of related ester analogs were next synthesized because they better mimic the parent carboxylic acid and thus might be less detrimental. An ester-linked 2-(dimethylamino)ethyl analog (6), similar to the amide-coupled amine 5c, displayed a 5-fold decrease in potency but only toward TBK1. To test whether the length of the modification could underlie the observed decrease in potency, a short ethyl ester (3) analog was synthesized, which decreased potency by more than 65 -fold toward TBK1 and 10-fold toward IKKe.

Carboxylic Acid Bioisosteres Improve Potency. Given the decrease in potency resulting from the elimination or derivatization of the carboxylic acid group, a series of bioisosteres was next investigated. Analogs of amlexanox containing tetrazole $(\mathbf{9})$, oxadiazolone $(\mathbf{1 1})$, or oxadiazole (12) groups in place of the carboxylic acid were synthesized. The tetrazole analog $\mathbf{9}$ displayed improved potencies of 400 and $200 \mathrm{nM}$ against TBK1 and $\mathrm{IKK} \varepsilon$, respectively. Despite reduced solubility (Table 2), installation of an oxadiazolone moiety (11) improved potency to $1.6 \mu \mathrm{M}$ toward IKK $\varepsilon$ but reduced potency toward TBK1 to $2.9 \mu \mathrm{M}$, consistent with IKK $\varepsilon$ being able to accept a wider variety of C-3 modifications. The oxadiazole analog 12 was essentially inactive against both TBK1 and IKK $\varepsilon$, likely because of its extremely poor aqueous solubility (Table 2 ).

Given the significant enhancement in potency of the tetrazole analog, an analog bearing a tetrazole attached to the core of amlexanox through an amide linkage, 5a, was 
TABLE 2

Solubility and Potencies of Amlexanox Analogs Against Kinase Variants

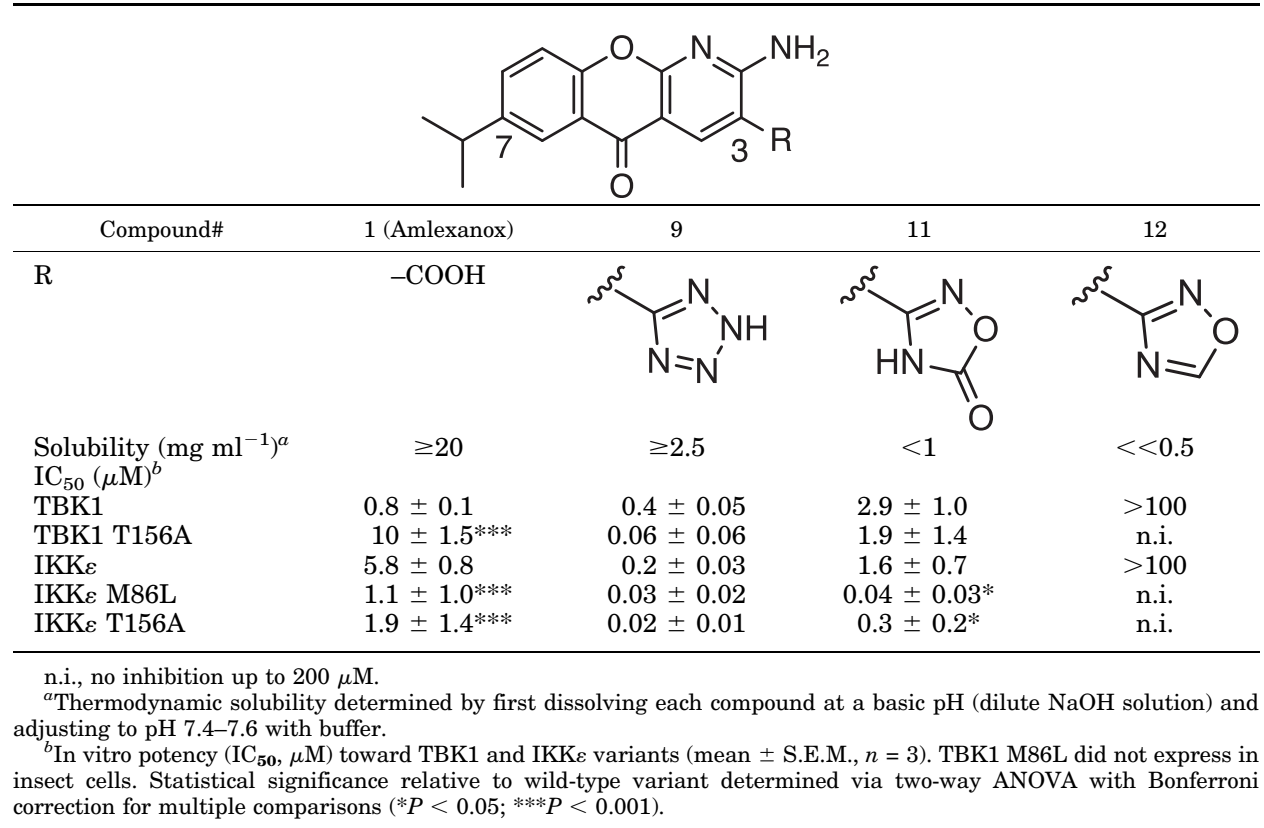

synthesized. 5a reduced potency 3 -fold toward TBK1 but improved potency by 3 -fold toward IKK $\varepsilon$.

Amlexanox Binds Along the Hinge of TBK1. In parallel with the preceding studies, an atomic structure of amlexanox in complex with TBK1 was determined to help interpret results and guide future SAR. Initial efforts to obtain a cocrystal structure with the better-expressing but inactive TBK1-K38A yielded no crystals, but differential scanning fluorometry with WT and TBK1-K38A revealed that the WT protein was stabilized, whereas the K38A variant was destabilized by amlexanox (Fig. 1A; Supplemental Fig. 1). Subsequently, the WT TBK1-amlexanox crystal structure was determined to $3.65 \AA$ spacings (PDB 5W5V, Fig. 1B; Supplemental Table 1 and Supplemental Material). The structure features strong density for amlexanox binding along the hinge of the kinase domain, mimicking the interactions made by the adenine ring of ATP. The binding mode is similar to that observed in the $\mathrm{G}$ protein-coupled receptor kinase 1-amlexanox complex (Homan et al., 2014).

The amine forms a hydrogen bond with the backbone carbonyl of Glu87, whereas the pyridine nitrogen interacts with the backbone nitrogen of Cys87. The side chain of Leu15 in the beginning of the phosphate-binding loop (P-loop) packs against the tricyclic core of the drug. The carboxylic acid on amlexanox is $4.1 \AA$ away from the hydroxyl of Thr156 and thus, considering the low resolution, forms either a weak hydrogen bond or favorable electrostatic interactions. Indeed, the TBK1-T156A variant displays a decrease in potency for amlexanox. Interestingly, amlexanox displays a slight increase in potency toward IKK $\varepsilon$-T156A (Table 2). No electron
A

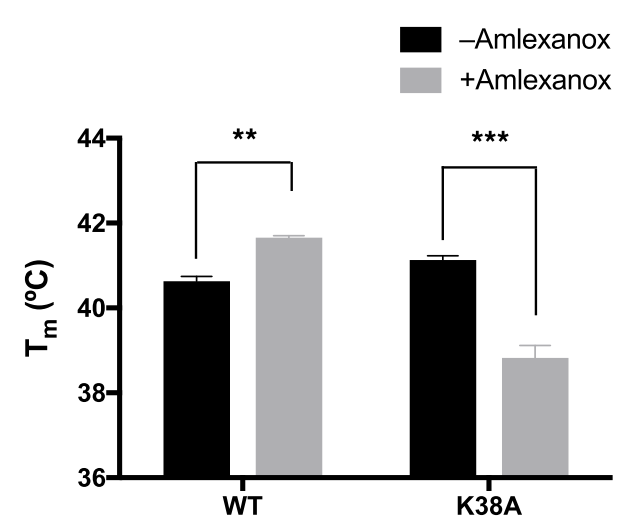

B

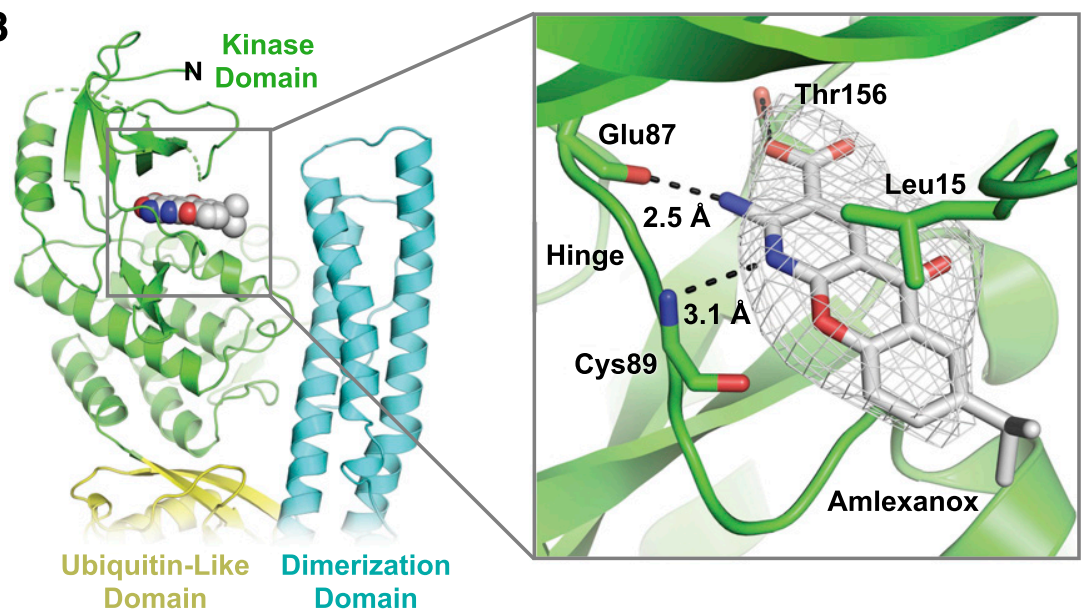

Fig. 1. Structural analysis of the TBK1 - amlexanox complex. (A) Amlexanox (200 $\mu \mathrm{M}$ ) stabilizes only the WT form of TBK1, as indicated by a positive shift in the melting temperature $T_{\mathrm{m}}$ (average \pm S.E.M., $n=2$, two-way ANOVA with Bonferroni correction for multiple comparisons to amlexanox sample. $* * P \leq 0.01 ; * * * P \leq 0.001$ ). (B) TBK1 - amlexanox crystal structure at $3.65 \AA$. Amlexanox (1) binds along the kinase hinge and forms specific interactions with the backbone of Glu87 and Cys89 and the side chain of Thr156. Mesh represents positive $\left|F_{\mathrm{o}}\right|-\left|F_{\mathrm{c}}\right|$ density contoured at $3.0 \sigma$. 
A

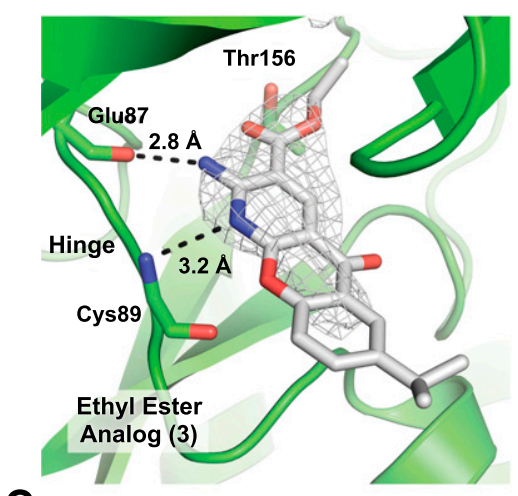

C

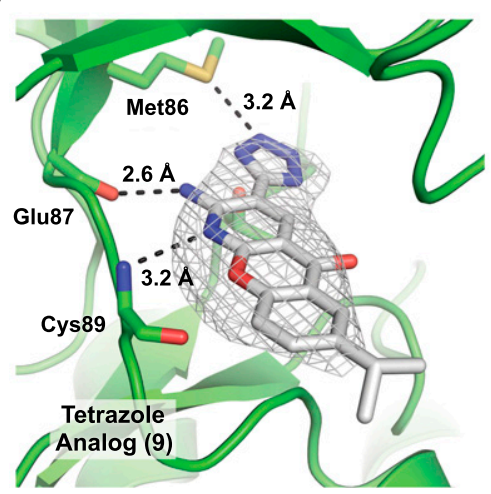

B

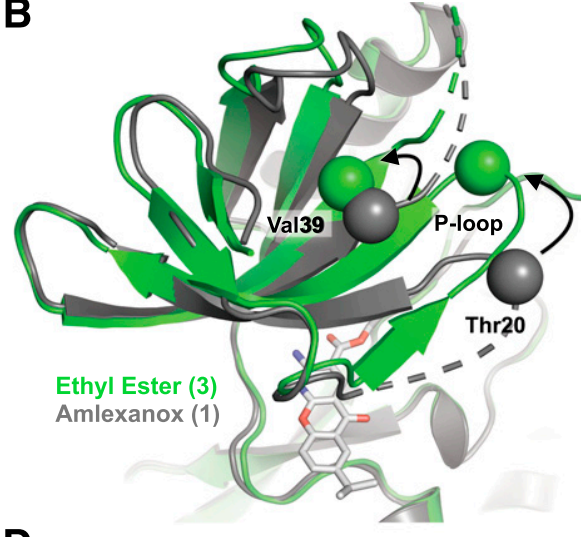

D

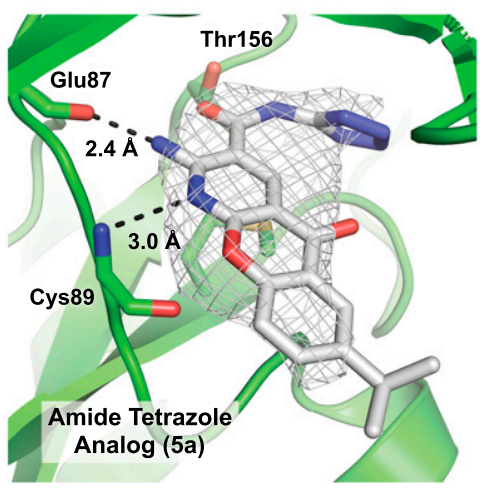

Fig. 2. Structural analysis of TBK1 in complex with carboxylic acid analogs of amlexanox. (A) TBK1 3 crystal structure at $3.2 \AA$. Mesh represents positive $\left|F_{\mathrm{o}}\right|-\left|F_{\mathrm{c}}\right|$ omit map density contoured at $2.0 \sigma$. (B) Alignment of the TBK1 . 3 (green) with TBK1 amlexanox (gray) complexes. The marked rotation in the P-loop is indicated by spheres at the $\mathrm{C} \alpha$ positions of Thr20 and Val39. (C) TBK1.9 crystal structure at $3.3 \AA$ with mesh representing $3.0 \sigma$ positive $\left|F_{\mathrm{o}}\right|-\left|F_{\mathrm{c}}\right|$ omit density. (D) TBK1.5a crystal structure at $3.6 \mathrm{~A}$ with mesh representing $3.0 \sigma$ positive $\left|F_{\mathrm{o}}\right|-\left|F_{\mathrm{c}}\right|$ omit density. Dashed black lines correspond to hydrogen bonds with corresponding distances indicated. density is observed for the side chain of Lys38, which was required for obtaining the cocrystal structure, indicating a high degree of mobility. Regardless, the Lys38 side chain would complement the negative charge of the carboxylate of amlexanox and, together with Thr156, help stabilize the compound in the active site.

Cocrystal Structures of Amlexanox C-3 Derivatives. To investigate the molecular basis for the loss in potency observed for $\mathbf{3}$ and $\mathbf{5 b}$, the crystal structure of TBK1 in complex with the ethyl ester analog 3 was determined to $3.2 \AA$ spacings (PDB 6BOD) (Supplemental Material). Weak ligand density is observed, consistent with its higher $55 \mu \mathrm{M} \mathrm{IC}_{50}$ value, but the planar density is situated along the hinge of the kinase domain similarly to the parent compound amlexanox (Fig. 2A). A shift in the P-loop and neighboring strand (residues 37-42) in the $\mathrm{N}$-terminal lobe of the kinase domain is also observed, resulting in a more open active site (Fig. 2B). Compared with the TBK1-amlexanox structure, additional density is observed for residues 160-163 adjacent to the DFG motif of the kinase domain. Thus, it seems that steric clashes introduced by the addition of an ethyl group induce a twisting motion of the $\mathrm{N}$-lobe of the kinase domain that leads to a more open kinase conformation.

To investigate the binding mode of the tetrazole analog $\mathbf{9}$, its cocrystal structure was solved at 3.34 A spacings (PDB 6BNY) (Supplemental Material). The compound once again binds similarly to amlexanox (Fig. 2C). Compared with the structure with $\mathbf{3}$, the kinase domain of TBK1 adopts a more closed conformation. Unlike amlexanox, which forms a relatively long hydrogen bond with Thr156, the side chain of Thr156 rotates to accommodate the tetrazole ring, which allows a $3.2 \AA$ hydrogen bond to form between the sulfur of the gatekeeper residue Met86 and the tetrazole moiety. The TBK1 variant M86L, another common gatekeeper residue in Ser/Thr kinases, did not express in insect cells, but $\mathbf{9}$ displayed an increase in potency toward IKK $\varepsilon$ M86L (Table 2). The related compound 11 displays increased potency toward IKK $\varepsilon$ M86L. Additionally, $\mathbf{9}$ and $\mathbf{1 1}$ display higher potencies against both TBK1 T156A and IKK $\varepsilon$ T156A (Table 2). A simple explanation for the enhanced potencies of these analogs toward these mutants is a general reduction of steric clashes with either Met86 or Thr156 that allows for improved packing of the cyclic bioisosteres.

Because other amide derivatives were significantly less potent than amlexanox, the molecular basis for the retained potency of 5 a was independently assessed. The $3.6 \AA$ co-crystal structure with TBK1 (PDB 6BOE) (Supplemental Material) indicates that the core of the molecule again binds along the hinge (Fig. 2D). Weak positive $\left|F_{\mathrm{o}}\right|-\left|F_{\mathrm{c}}\right|$ omitting map density is observed for the amide linker and terminal tetrazole ring. The amide arm assumes a cis conformation relative to the amide bond and core of the inhibitor and packs between Met142 and Val23. Residues 18-21 in the P-loop are disordered, disrupting P-loop crystal contacts observed in the other reported TBK1 structures and possibly accounting for the decreased resolution of the diffraction data. The loss in carboxylate interactions with Thr156 and Lys38 and disordering of the P-loop turn may be partially compensated for by favorable packing of the planar tetrazole ring under the ordered portion of the P-loop.

Bioisostere Analogs Display Limited Efficacy in Cells. To determine whether carboxylic acid modifications improve cellular efficacy, 3T3-L1 adipocytes were treated with the highest-potency tetrazole analog $(\mathbf{9})$ and the related 
A
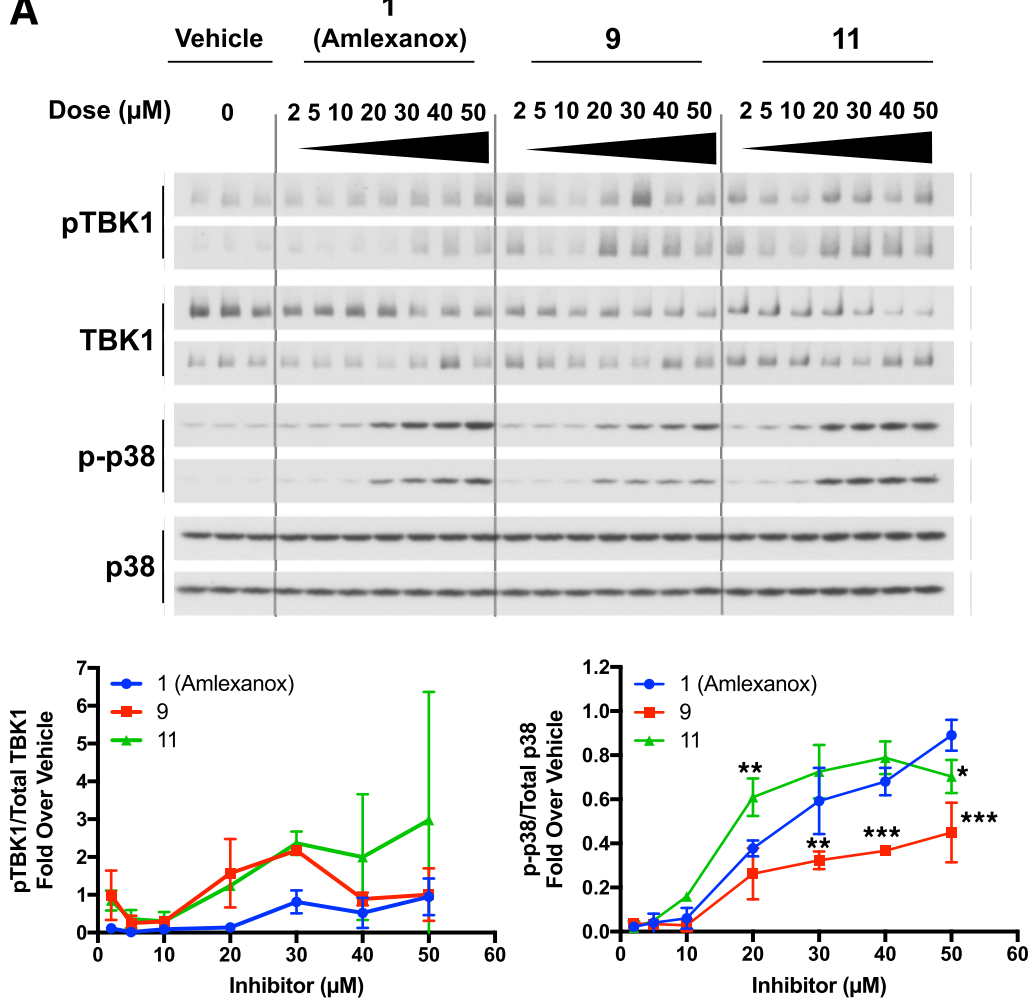

B

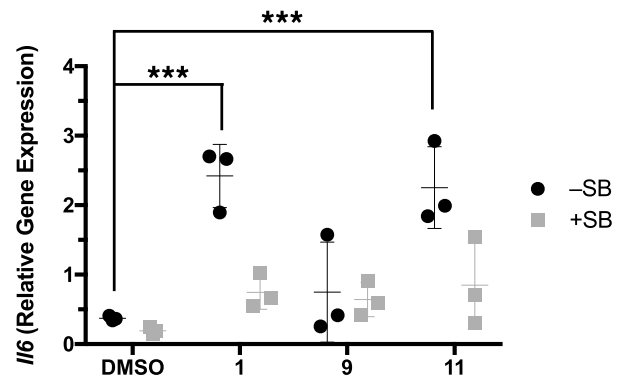

C

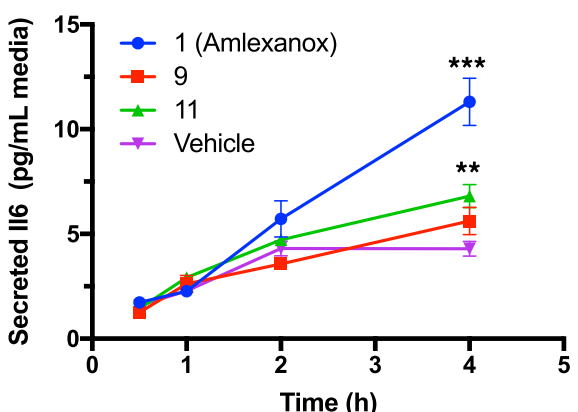

Fig. 3. Adipocyte cellular responses upon treatment with amlexanox (1) and selected analogs. (A) Western blot for phosphorylation of TBK1 and p38 MAPK after incubation of 3T3-L1 adipocytes with 2-50 $\mu \mathrm{M}$ amlexanox (1), 9, or 11 for 1 hour. Quantified results for pTBK1 and p-p38 are displayed below the blot (average \pm S.D., $n=2$ technical replicates, $* P \leq 0.05 ; * * \leq 0.01 ; * * * P \leq 0.001$, two-way ANOVA with Dunnett's correction for multiple comparisons relative to amlexanox). (B) Expression of $I l 6$ as determined by qPCR in 3T3-L1 adipocytes treated with $50 \mu \mathrm{M}$ compound for 4 hours. Expression was quantified with and without pretreatment with the p38 MAPK inhibitor SB203580 (average \pm S.D., $n=3$ biologic replicates, two-way ANOVA with Dunnett's correction for multiple comparisons relative to DMSO control, *** $P \leq 0.001$ ). (C) Secreted Il-6 from 3T3-L1 adipocytes treated with $50 \mu \mathrm{M}$ compound for 4 hours as determined by ELISA (average \pm S.D., $n=2$ technical replicates, two-way ANOVA with Dunnett's correction for multiple comparisons relative to DMSO control, **P $\leq 0.01$; ***P $\leq 0.001$ ).

oxadiazolone bioisostere (11). TBK1 mediates negative feedback regulation of AMP-activated protein kinase (AMPK), whereas IKK $\varepsilon$, and, to a lesser extent, TBK1 directly activate phosphodiesterase $3 \mathrm{~B}$ and thereby reduce intracellular cAMP levels to attenuate catecholamine-stimulated adrenergic receptor signaling. Thus, inhibition of both kinases produces a unique readout consisting of increased TBK1 phosphorylation at Ser172 as a consequence of the AMPK-mediated negative feedback loop (Clark et al., 2009; Clark et al., 2011; Reilly et al., 2013; Zhao et al., 2018), phosphorylation and activation of p38 MAPK (Reilly et al., 2015), and production of IL-6 (Reilly et al., 2015) downstream of cAMP signaling. Treatment of adipocytes with $\mathbf{9}$ or $\mathbf{1 1}$ simultaneously stimulated TBK1 and p38 phosphorylation in a dose-dependent manner (Fig. 3A). However, neither 9 nor 11 significantly increased pTBK1 response compared with amlexanox. Furthermore, although 11 more potently induces p38 phosphorylation, the maximum response is not greater than that of amlexanox. Compound 9 was less effective than amlexanox at stimulating p38 phosphorylation, likely resulting from decreased permeability, assessed by cLogP (Table 1), and poor compound solubility in the assay medium (Table 2). Adipocytes treated with amlexanox or 11 display significantly increased $I l 6$ expression (Fig. 3B). In agreement with previous studies, induction of $I l 6$ is blocked by pretreatment with the p38 MAPK inhibitor SB203580, confirming that the observed response to $\mathbf{1 1}$ is through activation of p38 (Barancík et al., 2001; Reilly et al., 2015). The tetrazole analog 9 did not induce $I l 6$ gene expression. Secretion of IL-6 from treated adipocytes was also assessed by ELISA (Fig. 3C). In line with $I l 6$ expression, only treatment with analog $\mathbf{1 1}$ resulted in a significant increase in IL-6 secretion, although the increase was less robust than that produced by amlexanox.

\section{Discussion}

Amlexanox shows promise as a therapeutic lead for the treatment of obesity and type 2 diabetes via inhibition of kinases that are effectors of chronic low-grade inflammation in hepatic and adipose tissues. Initial characterization showed inhibition by amlexanox to be through an ATP-competitive mechanism; however, the precise binding mode and molecular interactions leading to inhibition were unknown (Reilly, et al., 2013). The structure of TBK1 bound to amlexanox reveals canonical type I ATP-competitive binding along the hinge of the kinase in the space occupied by the adenine ring of ATP. Compared with previously reported TBK1 structures in complex with the nanomolar inhibitors BX795 and MRT67307 (Ma et al., 2012; Larabi et al., 2013; Tu et al., 2013), the small lobe of the kinase domain appears less ordered when in complex with amlexanox, particularly residues $15-19$ in the P-loop. This finding was not unexpected because amlexanox is 
a lower potency inhibitor and occupies only the region along the hinge, whereas the more extended BX795 and MRT67307 inhibitors also pack under the P-loop.

The interaction between the carboxylate on amlexanox and the side chain hydroxyl of Thr156 amine is important for the inhibition of TBK1, but less so in the context of IKK $\varepsilon$, where removal of the carboxylic acid moiety in $\mathbf{7}$ or introduction of the T156A mutation instead significantly improves potency. Reduction of the carboxylic acid to a primary alcohol in $\mathbf{4}$ also only perturbs inhibition of TBK1. As TBK1 and IKKe are $>70 \%$ identical in their kinase domains, the improved potency exhibited toward IKK $\varepsilon$ by $\mathbf{7}$ and $\mathbf{5 b}$ is surprising. One notable difference between the two kinases lies at position 90 in the hinge, which corresponds to a proline in TBK1 and a serine in IKKe. The presence of a proline in the hinge of TBK1 may restrict the relative orientation of its kinase domain lobes. Thus, decreased potency may selectively occur in TBK1 because it is less able to reconfigure itself and optimally interact with inhibitors lacking a carboxylate. Verification of this model will have to await determination of atomic structures of IKK $\varepsilon$. Disruption of the carboxylate-Thr156 interaction, however, seems to be a viable strategy for improving selectivity, and often potency, toward IKK $\varepsilon$.

All amide and ester modifications of the carboxylic acid of amlexanox decreased potency toward TBK1, whereas only $\mathbf{5 d}$ significantly decreased potency toward IKK $\varepsilon$. Given the effect of TBK1- and IKK $\varepsilon$-T156A on amlexanox potency, the trend may simply arise from disruption of the carboxylate-Thr156 hydroxyl interaction. Extensions to the carboxylic acid introduce steric clashes in TBK1 that further impede ligand binding. Indeed, the cocrystal structure of TBK1 bound to 3 reveals that esterification of amlexanox does not change the binding position of the core of the inhibitor along the kinase hinge but leads to a twist in the P-loop and a more open conformation of the kinase domain, which may stem from clashes between the ethyl modification and the kinase DFG motif and P-loop. The amide- and ester-coupled amines 5c and 6, respectively, and the short methyl amide 5b, display no significant reduction in potency toward IKK $\varepsilon$, possibly as a result of increased hinge flexibility in this enzyme relative to TBK1. The coupling of a 5-membered tetrazole ring through an amide linkage in $\mathbf{5 a}$ improves IKK $\varepsilon$ inhibition but exhibits only a modest decrease in potency toward TBK1, whereas the 6-membered 2-morpholinoethyl analog $\mathbf{5 d}$ is a poor inhibitor of both kinases. These results further suggest that there is a steric size limit for packing under the P-loop. Indeed, other potent but promiscuous TBK1 and IKK $\varepsilon$ inhibitors, such as BX795 and MRT67307, pack 4- and 5-membered cyclobutyl and thiophene rings under the P-loop (Ma et al., 2012; Larabi et al., 2013; Tu et al., 2013).

The gatekeeper residue in kinases plays a key role in the formation of hydrophobic spines that must form for the kinase to assume and active conformation (Taylor and Kornev, 2011). Therefore, Met86 was mutated to leucine, another common Ser/Thr kinase gatekeeper residue, to minimally perturb kinase activity and conformation. Surprisingly, the M86L variant of TBK1 did not express, whereas IKKe M86L displayed increased potency toward amlexanox (1), 9, and 11, despite the loss of a hydrogen bond between Met86 and the tetrazole ring of $\mathbf{9}$ and no interactions between Met86 and amlexanox. Because amlexanox and analogs thereof are type
1 inhibitors that favor binding to the active conformation of kinases, it is possible that M86L better stabilizes the kinase hydrophobic spine leading to a more active kinase conformation and improved potencies or simply enlarged the binding pocket such that the bulkier acid bioisosteres are better accommodated.

Because TBK1 and IKKe play different roles in chronic inflammation associated with obesity (Chiang et al., 2009; Zhou et al., 2018), the development of inhibitors selective for either kinase would enable pharmacologic interrogation of the signaling pathways associated with each enzyme. The SAR around the carboxylic acid moiety of amlexanox reveals that $\mathrm{IKK} \varepsilon$ is more tolerant to modifications at the C-3 position. Simple decarboxylation of amlexanox (7) improves selectivity toward IKK $\varepsilon$ by more than one order of magnitude. Other modifications, such as conversion of the carboxylic acid to a methyl amide (5) $)$, also improve selectivity toward IKK $\varepsilon$. Coupled with modifications at other positions, such as the C-7 isopropyl, these and related carboxylic acid derivatives represent possible routes to the generation of IKK $\mathrm{I}$-selective inhibitors.

The lack of improved cellular activity observed for the tetrazole analog of amlexanox is not surprising, as tetrazole modifications have been reported to decrease cellular permeability (Table 1) (Lassalas et al., 2016). Despite a decrease in potency toward TBK1 and solubility (Table 2), the oxadiazolone analog $\mathbf{1 1}$ produces a significant cellular response, albeit less than amlexanox, perhaps owing to increased cellular permeability $(\mathrm{cLogP})$ of oxadiazolones compared with carboxylic acids (Table 1) (Lassalas et al., 2016). Because amlexanox and related analogs are type 1 kinase inhibitors with a general ATP-competitive mechanism, we cannot exclude the possibility of off-target effects, especially given the indirect nature of measuring $\mathrm{p}-\mathrm{TBK} 1$, p-p38 MAPK, and IL-6; however, this particular combination of readouts has been extensively validated in the context of amlexanox (Reilly et al., 2013, 2015; Zhao et al., 2018). Given the modest nature of the C-3 modifications and observed effects in all three readouts, analogs $\mathbf{9}$ and $\mathbf{1 1}$ are likely on-target, at least in the context of cultured adipocytes. The selectivity of analogs exhibiting more promising cellular efficacy should be thoroughly assessed before studies in vivo to predict and minimize deleterious off-target effects stemming from inhibition of kinases prevalent in other cell types.

Because free carboxylic acid groups are prone to glucuronidation during phase 2 metabolism (Sakaguchi et al., 2004), installation of less acidic bioisosteres may improve metabolic properties of amlexanox; however, in assays with cultured adipocytes, metabolism is expected to be insignificant because of a lack of hepatic drug-metabolizing enzymes. Difficulties solubilizing 9 and 11 (Table 2) prohibited testing in obese mice and highlights another pharmacokinetic property that may be improved through the installation of alternative bioisosteres. The cocrystal structure with the tetrazole analog 9 will serve as a guide toward identifying alternative bioisosteres that maintain or improve potency while also improving cellular response. In particular, the related thiadiazolone and isoxazole bioisosteres are predicted to be significantly more cell permeable and are options for future exploration (Vitaku et al., 2014; Lassalas et al., 2016). 


\section{Acknowledgments}

We thank David Ginsburg (University of Michigan) for the qPCR instrument used for thermal shift experiments.

\section{Authorship Contributions}

Participated in research design: Beyett, Gan, Reilly, Chang, Saltiel, Showalter, Tesmer.

Conducted experiments: Beyett, Gan, Reilly, Chang, Gomez.

Contributed new reagents of analytic tools: Gan.

Performed data analysis: Beyett, Gan, Reilly, Chang, Gomez, Saltiel, Showalter, Tesmer.

Wrote or contributed to the writing of the manuscript: Beyett, Gan, Saltiel, Showalter, Tesmer.

\section{References}

Adams PD, Afonine PV, Bunkóczi G, Chen VB, Davis IW, Echols N, Headd JJ, Hung L-W, Kapral GJ, Grosse-Kunstleve RW, et al. (2010) PHENIX: a comprehensive Python-based system for macromolecular structure solution. Acta Crystallogr D Biol Crystallogr 66:213-221.

Barancík M, Bohácová V, Kvackajová J, Hudecová S, Krizanová O, and Breier A (2001) SB203580, a specific inhibitor of p38-MAPK pathway, is a new reversal agent of P-glycoprotein-mediated multidrug resistance. Eur J Pharm Sci 14:29-36

Bell J (2005) Amlexanox for the treatment of recurrent aphthous ulcers. Clin Drug Investig 25:555-566.

Chen VB, Arendall WB III, Headd JJ, Keedy DA, Immormino RM, Kapral GJ, Murray LW, Richardson JS, and Richardson DC (2010) MolProbity: all-atom structure validation for macromolecular crystallography. Acta Crystallogr D Biol Crystallogr 66:12-21.

Chiang S-H, Bazuine M, Lumeng CN, Geletka LM, Mowers J, White NM, Ma J-T, Zhou J, Qi N, Westcott D, et al. (2009) The protein kinase IKKepsilon regulates energy balance in obese mice. Cell 138:961-975.

Clark K, Peggie M, Plater L, Sorcek RJ, Young ERR, Madwed JB, Hough J, McIver EG, and Cohen P (2011) Novel cross-talk within the IKK family controls innate immunity. Biochem J 434:93-104.

Clark K, Plater L, Peggie M, and Cohen P (2009) Use of the pharmacological inhibitor BX795 to study the regulation and physiological roles of TBK1 and IkappaB kinase epsilon: a distinct upstream kinase mediates Ser-172 phosphorylation and activation. J Biol Chem 284:14136-14146.

Emsley P, Lohkamp B, Scott WG, and Cowtan K (2010) Features and development of Coot. Acta Crystallogr D Biol Crystallogr 66:486-501.

Homan KT, Wu E, Cannavo A, Koch WJ, and Tesmer JJ (2014) Identification and characterization of amlexanox as a $\mathrm{G}$ protein-coupled receptor kinase 5 inhibitor. Molecules 19:16937-16949.

Kabsch W (2010) XDS. Acta Crystallogr D Biol Crystallogr 66:125-132.

Larabi A, Devos JM, Ng S-L, Nanao MH, Round A, Maniatis T, and Panne D (2013) Crystal structure and mechanism of activation of TANK-binding kinase 1. Cell Rep 3:734-746.

Lassalas P, Gay B, Lasfargeas C, James MJ, Tran V, Vijayendran KG, Brunden KR, Kozlowski MC, Thomas CJ, Smith AB III, et al. (2016) Structure property relationships of carboxylic acid isosteres. J Med Chem 59:3183-3203.
Ma X, Helgason E, Phung QT, Quan CL, Iyer RS, Lee MW, Bowman KK, Starovasnik MA, and Dueber EC (2012) Molecular basis of tank-binding kinase 1 activation by transautophosphorylation. Proc Natl Acad Sci USA 109:9378-9383.

Makino H, Saijo T, Ashida Y, Kuriki H, and Maki Y (1987) Mechanism of action of an antiallergic agent, amlexanox (AA-673), in inhibiting histamine release from mast cells: acceleration of cAMP generation and inhibition of phosphodiesterase. Int Arch Allergy Appl Immunol 82:66-71.

McCoy AJ, Grosse-Kunstleve RW, Adams PD, Winn MD, Storoni LC, and Read RJ (2007) Phaser crystallographic software. J Appl Cryst 40:658-674.

Moriarty NW, Grosse-Kunstleve RW, and Adams PD (2009) Electronic Ligand Builder and Optimization Workbench (eLBOW): a tool for ligand coordinate and restraint generation. Acta Crystallogr D Biol Crystallogr 65:1074-1080.

Morin A, Eisenbraun B, Key J, Sanschagrin PC, Timony MA, Ottaviano M, and Sliz P (2013) Collaboration gets the most out of software. eLife 2:e01456.

Mowers J, Uhm M, Reilly SM, Simon J, Leto D, Chiang S-H, Chang L, and Saltiel AR (2013) Inflammation produces catecholamine resistance in obesity via activation of PDE3B by the protein kinases IKK $\varepsilon$ and TBK1. eLife 2:e01119.

Nohara A, T. Ishiguro T, Ukawa K, Sugihara H, Maki Y, and Sanno Y (1985) Studies on antianaphylactic agents. 7. Synthesis of antiallergic 5-oxo-5H-[1]benzopyrano [2,3-b]pyridines. J Med Chem 28:559-568.

Oral EA, Reilly SM, Gomez AV, Meral R, Butz L, Ajluni N, Chenevert TL, Korytnaya E, Neidert AH, Hench R, et al. (2017) Inhibition of IKK and TBK1 improves glucose control in a subset of patients with type 2 diabetes. Cell Metab 26:157-170.e7.

Reilly SM, Ahmadian M, Zamarron BF, Chang L, Uhm M, Poirier B, Peng X, Krause DM, Korytnaya E, Neidert A, et al. (2015) A subcutaneous adipose tissue-liver signalling axis controls hepatic gluconeogenesis. Nat Commun 6:6047.

Reilly SM, Chiang S-H, Decker SJ, Chang L, Uhm M, Larsen MJ, Rubin JR, Mowers J, White NM, Hochberg I, et al. (2013) An inhibitor of the protein kinases TBK1 and IKKimproves obesity-related metabolic dysfunctions in mice. Nat Med 19:313-321.

Reilly SM and Saltiel AR (2017) Adapting to obesity with adipose tissue inflammation. Nat Rev Endocrinol 13:633-643.

Sakaguchi K, Green M, Stock N, Reger TS, Zunic J, and King C (2004) Glucuronidation of carboxylic acid containing compounds by UDP-glucuronosyltransferase isoforms. Arch Biochem Biophys 424:219-225.

Saltiel AR (2016) New therapeutic approaches for the treatment of obesity. Sci Transl Med 8:323rv2.

Swinburn BA, Sacks G, Hall KD, McPherson K, Finegood DT, Moodie ML, and Gortmaker SL (2011) The global obesity pandemic: shaped by global drivers and local environments. Lancet 378:804-814.

Taylor SS and Kornev AP (2011) Protein kinases: evolution of dynamic regulatory proteins. Trends Biochem Sci 36:65-77.

Tu D, Zhu Z, Zhou AY, Yun C-H, Lee K-E, Toms AV, Li Y, Dunn GP, Chan E, Thai T, et al. (2013) Structure and ubiquitination-dependent activation of TANK-binding kinase 1. Cell Rep 3:747-758.

Vitaku E, Smith DT, and Njardarson JT (2014) Analysis of the structural diversity, substitution patterns, and frequency of nitrogen heterocycles among U.S. FDA approved pharmaceuticals. J Med Chem 57:10257-10274.

Zhao P, Wong KI, Sun X, Reilly SM, Uhm M, Liao Z, Skorobogatko Y, and Saltiel AR (2018) TBK1 at the crossroads of inflammation and energy homeostasis in adipose tissue. Cell 172:731-743.e12.

Address correspondence to: Dr. John J. G. Tesmer, Purdue University, 240 S. Martin Jischke Drive, Room 329, West Lafayette, IN 47907-2054. E-mail: jtesmer@purdue.edu 